\title{
Clinical Factors Associated with Initial Decrease in Body-Fat Percentage Induced by Add-on Sodium-Glucose Co-transporter 2 Inhibitors in Patient with Type 2 Diabetes Mellitus
}

\author{
Noboru Kurinami ${ }^{1}$-Seigo Sugiyama ${ }^{1,2} \cdot$ Hiroyuki Nishimura ${ }^{1} \cdot$ Ayami Morita $^{1}$. \\ Akira Yoshida $^{1}$ - Kunio Hieshima ${ }^{1}$ - Fumio Miyamoto ${ }^{1}$ - Keizo Kajiwara ${ }^{1}$. \\ Katsunori Jinnouchi ${ }^{1} \cdot$ Tomio Jinnouchi $^{1} \cdot$ Hideaki Jinnouchi ${ }^{1}$
}

Published online: 2 November 2017

(C) The Author(s) 2017. This article is an open access publication

\begin{abstract}
Background and objective Obesity is globally recognized as an important clinical problem and sodium-glucose cotransporter 2 (SGLT2) inhibitors are considered a suitable therapy for obese patients with type 2 diabetes mellitus (T2DM). We examined the clinical factors associated with initial decrease in body-fat percentage (Fat \%) induced by SGLT2 inhibitors in patients with T2DM.

Methods We retrospectively enrolled patients newly treated with SGLT2 inhibitors in addition to ongoing medications at Jinnouchi Hospital between April 2014 and December 2015. We examined the SGLT2 inhibitor-induced change in body composition by using a bioelectrical impedance analyzer $\left(\operatorname{InBody} 770^{\circledR}\right)$ before SGLT2 inhibitor administration and after 4 weeks' treatment.

Results A total of 175 patients with T2DM were enrolled and we analyzed 148 patients. Add-on SGLT2 inhibitor treatment significantly reduced body weight $(-1.04 \pm$ $1.18 \mathrm{~kg}, p<0.01)$, total fat quantity $(-0.62 \pm 1.19 \mathrm{~kg}$, $p<0.01)$, and Fat $\%(-0.4 \pm 1.4 \%, p<0.01)$. Pretreatment levels of glycated hemoglobin (HbA1c) [odds ratio (OR), 1.61; 95\% confidence interval (CI), 1.15-2.25, $p<0.01]$ and smoking (OR, 2.65; 95\% CI, 1.14-6.15, $p=0.02$ ) were significantly associated factors for greater fat-reduction defined as more than $0.4 \%$ (median) decrease
\end{abstract}

Noboru Kurinami and Seigo Sugiyama contributed equally to this study.

Hideaki Jinnouchi

hideaki@jinnouchi.or.jp

1 Diabetes Care Center, Jinnouchi Hospital, 6-2-3 Kuhonji, Chuo-ku, Kumamoto 862-0976, Japan

2 Division of Cardiovascular Medicine, Diabetes Care Center, Jinnouchi Hospital, Kumamoto, Japan in Fat $\%$ in multivariate logistic regression analysis. In receiver operator characteristic analysis, the cut-off value of pretreatment levels of $\mathrm{HbAlc}$ for a greater Fat $\%$ decrease was $7.7 \%$ (sensitivity $53 \%$ and specificity $69 \%$, $p<0.01$ ).

Conclusion Additional treatment with SGLT2 inhibitors effectively decreased Fat \% in T2DM patients with high $\mathrm{HbA} 1 \mathrm{c}$ levels before SGLT2 inhibitor administration. Our results suggest a greater initial response in Fat \% reduction to SGLT2 inhibitor therapy in diabetic patients with pretreatment $\mathrm{HbA1c}$ levels $\geq 7.7 \%$.

\section{Key Points}

Add-on SGLT2 inhibitor treatment significantly reduced body weight, total fat quantity, and body-fat percentage.

Pretreatment $\mathrm{HbA} 1 \mathrm{c}$ levels and smoking were significantly associated with greater body fat reduction with SGLT2 inhibitors.

Reduction in body-fat percentage was significantly greater in the higher HbA1c group (pretreatment HbA1c levels $\geq 7.7 \%$ ) than the lower HbA1c group (pretreatment HbA1c levels $<7.7 \%$ ), but the reduction in body weight was not significantly different between the two groups. 


\section{Introduction}

It has been well established that obesity is closely involved in the pathogenesis of type 2 diabetes mellitus (T2DM). At present, the incidence of obesity is continually increasing and is globally recognized as an important clinical problem [1]. In patients with T2DM, diabetes treatment accompanied by body fat and weight reduction may become a valuable strategy. This would provide an additional beneficial effect on the fundamental pathophysiology of T2DM.

Sodium-glucose co-transporter 2 (SGLT2) inhibitors inhibit the reabsorption of glucose in the kidney. It is expected that there will be an insulin-independent improvement of hyperglycemia through the promotion of urinary glucose excretion and enhancement of lipolysis with a reduction in glucose incorporation into the somatic cells. A previous report demonstrated that dapagliflozin has an antidiabetic action equivalent to glipizide [2]. In addition, a meta-analysis reported that weight decreases by $1 \mathrm{~kg}$ and body fat decreases by $2.73 \%$ with SGLT2 inhibitors compared to placebo [3]. Furthermore, weight reduction by dapagliflozin is reported to be mainly due to fat reduction [4]. SGLT2 inhibitors do not have a labelled indication for weight loss, but SGLT2 inhibitors have exhibited weight reduction during treatment of T2DM. Therefore, SGLT2 inhibitors are considered a suitable therapy for obese patients with T2DM. However, the clinical factors associated with effective weight and fat reduction are unknown.

The weight-reducing effect of SGLT2 inhibitors is achieved within 6 months from first administration [5]. It has been reported that ipragliflozin treatment decreased body weight by $1.4 \pm 0.5 \mathrm{~kg}$ in 7 days [6]. To achieve the initial effectiveness of the SGLT2 inhibitor antidiabetic treatment for T2DM patients, we need to know the baseline clinical factors associated with SGLT2 inhibitor-induced body fat reduction.

In this study, based on results obtained from treatment with add-on SGLT2 inhibitors for glucose reduction of T2DM, we examined the clinical factors associated with initial decrease in Fat \% induced by add-on SGLT2 inhibitors in patients with T2DM.

\section{Methods}

\subsection{Subjects and Protocol}

Patients with T2DM who visited the Diabetes Care Center of Jinnouchi Hospital between April 2014 and December 2015 and were treated with SGLT2 inhibitors in addition to their ongoing medications were retrospectively registered. We measured body composition using a body composition analyzer (InBody770; Biospace, Seoul, Korea) both before administration of SGLT2 inhibitors and 4 weeks after treatment and calculated the change in composition. Written informed consent was obtained from all patients. The study was conducted in accordance with the Declaration of Helsinki and the Human Ethics Review Committee of Jinnouchi Hospital approved the study protocol.

\subsection{Measurement of Body Fat and Muscle Composition}

Elementary body composition was measured using a direct, segmental, multi-frequency bioelectrical impedance analyzer (InBody770; Biospace, Seoul, Korea). This analyzer processes 30 impedance measurements using six different frequencies $(1,5,50,250,500$, and $1000 \mathrm{kHz})$ at each of the five body segments (right and left arms, right and left legs, and trunk) and 15 reactance measurements using tetrapolar 8-point tactile electrodes at three different frequencies $(5,50$, and $250 \mathrm{kHz})$ at each of the same five body segments [7, 8]. Body composition analyses were conducted with the subjects clothed as specified by the hospital and performed once before starting SGLT2 inhibitors and again after 4 weeks of treatment.

\subsection{Blood Sampling and Measurement of Clinical Parameters}

Measurement of glycated hemoglobin (HbA1c) and plasma glucose is routinely performed every month in patients with diabetes mellitus (DM). Moreover, biochemical examinations of blood serum chemistry are usually performed before and after newly started medications including SGLT2 inhibitors to determine drug-induced side effects. Blood samples for HbAlc, total cholesterol, lowdensity lipoprotein (LDL) cholesterol, high-density lipoprotein (HDL) cholesterol, triglycerides, and estimated glomerular filtration ratio (eGFR) were collected from the antecubital vein before SGLT2 inhibitor therapy and analyzed in the Jinnouchi Hospital laboratory. In our clinic body composition in all patients starting SGLT2 inhibitors is routinely measured at initiation and after 4 weeks of SGLT2 inhibitor therapy.

\subsection{Statistical Analysis}

The Shapiro-Wilk test was used to assess the normal distribution of continuous data. Data were expressed as mean \pm standard deviation (SD), and data with skewed distributions were expressed as the median value with interquartile range. Categorical data were presented as frequencies and percentages. Differences between paired samples were analyzed by the $t$ test or Wilcoxon signed- 
rank test; the differences between two groups were analyzed by the $t$ test, Mann-Whitney $U$ test, or Fisher's exact test as appropriate. Add-on SGLT2 inhibitor treatment significantly reduced body weight, total fat quantity, and Fat $\%$. However, body weight change and body fat quantity change did not show a strong correlation. Therefore, we used Fat $\%$ as the effective parameter in this study. Calculation of the Pearson product-moment correlation coefficient was used to examine the relationships between the weight change with SGLT2 inhibitors and the amount of Fat $\%$ change. Multivariate regression analysis was conducted using the significant outcomes by the Pearson product-moment correlation analysis, age, sex, and body mass index (BMI). Median Fat \% reduction after add-on SGLT2 inhibitor treatment was $-0.4 \%$. Thus we defined a greater response in fat reduction following SGLT2 inhibitors as more than $0.4 \%$ reduction in Fat \%, which we analyzed using simple logistic regression analysis with the greater fat reduction as the dependent variable and the various parameters as independent variables. To investigate the clinically associated factors with the greater body fat reduction following SGLT2 inhibitor medication, multivariate logistic regression analysis was conducted among the significant factors of simple logistic regression analysis. The Hosmer-Lemeshow test was conducted to investigate the goodness of fit of the logistic regression model. Receiver-operating characteristic (ROC) curve analysis was conducted to calculate a cut-off value of pretreatment levels of $\mathrm{HbA1c}$ for greater body fat reduction. A p-value $<0.05$ denoted statistical significance. Statistical analyses were performed using SPSS version 23 (SPSS Inc., Tokyo, Japan).

\section{Results}

\subsection{Subjects}

A total of 175 patients with T2DM were enrolled and all patients had body composition measured at the start of the SGLT2 inhibitor add-on therapy, but we were unable to measure body composition in 27 patients at the end of 4 weeks. Thus, 27 patients were excluded. Table 1 shows the baseline characteristics of the participants. SGLT2 inhibitors were newly added to the ongoing medications as follows: dapagliflozin $(n=46,30.9 \%)$, tofogliflozin $(n=45,30.4 \%)$, luseogliflozin $(n=35,23.6 \%)$, ipragliflozin $(n=20,13.5 \%)$, and empagliflozin $(\mathrm{n}=2,1.4 \%)$. Combined antidiabetic medications were insulin $(n=44$, 29.7\%), glucagon-like peptide-1 (GLP-1) receptor analog $(n=13,8.8 \%)$, sulfonylurea $(n=63,42.6 \%)$, dipeptidyl peptidase 4 inhibitor $(n=48,32.4 \%)$, metformin $(n=71$, $48.0 \%)$, thiazolidine $(n=15,10.1 \%)$, glinide $(n=12$,
Table 1 Clinical characteristics of the study participants

\begin{tabular}{ll}
\hline Characteristic & Value \\
\hline Males & $107(72.3)$ \\
Age (year) & $58(50-63)$ \\
Height $(\mathrm{cm})$ & $165.9 \pm 8.2$ \\
Weight $(\mathrm{kg})$ & $76.4(66.0-85.5)$ \\
BMI $\left(\mathrm{kg} / \mathrm{m}^{2}\right)$ & $27.5(25.1-30.2)$ \\
Waist circumference $(\mathrm{cm})$ & $95.5(88.2-103.2)$ \\
Muscle quantity $(\mathrm{kg})$ & $48.9 \pm 8.7$ \\
Body-fat quantity $(\mathrm{kg})$ & $25.0(18.1-30.3)$ \\
Body-fat percentage $(\%)$ & $32.3 \pm 8.0$ \\
Hypertension & $85(57.4)$ \\
Dyslipidemia & $127(85.8)$ \\
Current smoking & $39(26.4)$ \\
Hemoglobin A1c $(\%)$ & $7.5(7.0-8.2)$ \\
Total cholesterol $(\mathrm{mg} / \mathrm{dl})$ & $178.5 \pm 36.4$ \\
HDL-cholesterol $(\mathrm{mg} / \mathrm{dl})$ & $47.5(40.0-56.0)$ \\
LDL-cholesterol $(\mathrm{mg} / \mathrm{dl})$ & $96.0(76.0-120.3)$ \\
Triglycerides $(\mathrm{mg} / \mathrm{dl})$ & $121.0(86.5-194.5)$ \\
eGFR (ml/min/1.73 m $\left.{ }^{2}\right)$ & $71.2(62.2-83.2)$ \\
\hline
\end{tabular}

Data are mean \pm standard deviation (SD), median (25-75th percentile range), or number (\%)

$B M I$ body mass index, $L D L$ low-density lipoprotein, $H D L$ high-density lipoprotein, $e G F R$ estimated glomerular filtration rate

$8.1 \%)$, and alpha glucosidase inhibitor $(n=24,16.2 \%)$. Among the included patients, 85 (57.4\%) had hypertension and $127(85.4 \%)$ had dyslipidemia. They were treated with calcium antagonists $(n=63,42.6 \%)$, angiotensin II receptor blockers $(n=75,50.7 \%)$, angiotensin-converting enzyme inhibitors $(n=2,1.4 \%)$, beta blockers $(n=25$, $26.4 \%)$, statins $(n=76,51.4 \%)$, and ezetimibe $(n=18$, $12.2 \%)$.

\subsection{Change in Body Composition after Add-on SGLT2 Inhibitor Treatment}

Add-on SGLT2 inhibitor treatment for 4 weeks significantly reduced body weight $(-1.04 \pm 1.18 \mathrm{~kg}, p<0.01)$, total fat quantity $(-0.62 \pm 1.19 \mathrm{~kg}, p<0.01)$, and Fat $\%$ $(-0.4 \pm 1.4 \%, p<0.01)$. Figure 1 shows the scatter plot diagram of the changes in total body weight and body fat. The body fat changes were significantly correlated with the changes in total body weight, but the correlation was not strong $(r=0.44, p<0.001)$. In Pearson's correlation analysis, reduction in body fat quantity was not statistically significantly correlated with baseline body weight $(r=0.07, \quad p=0.38)$ and baseline BMI $(r=0.05$, $p=0.57)$. 


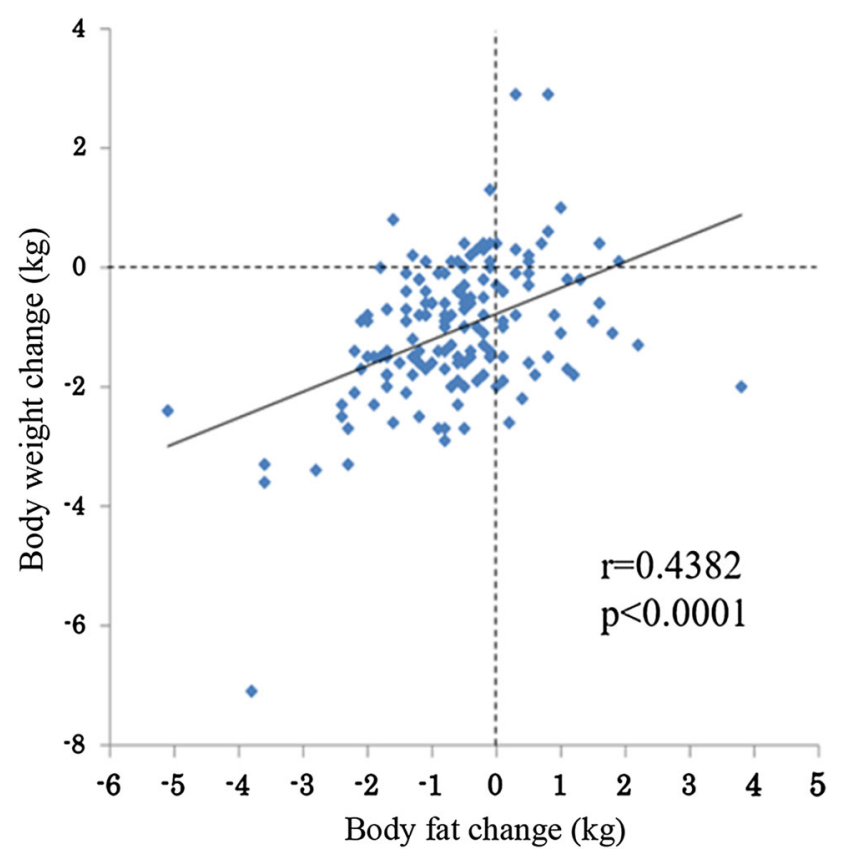

Fig. 1 Relationship between the amount of body weight change and body fat change following add-on SGLT2 inhibitor treatment $(r=0.44 ; p<0.0001)$. SGLT2 sodium-glucose co-transporter 2

\subsection{Pearson Product-Moment Correlation Analysis for Changes in Fat \% after Add-on SGLT2 Inhibitor Treatment}

Table 2 shows the results of the simple linear regression analysis conducted for changes in Fat $\%$ with various parameters. Pretreatment levels of HbA1c $(r=-0.20$, $p=0.015)$ and estimated eGFR $(r=-0.23, p=0.005)$

Table 2 Results of Pearson product-moment correlation analysis conducted for changes in body-fat percentage

\begin{tabular}{lrll}
\hline Variable & \multicolumn{1}{l}{$r$} & \multicolumn{1}{l}{$R^{2}$} & $p$ value \\
\hline Age (year) & 0.0948 & 0.0090 & 0.2517 \\
Height $(\mathrm{cm})$ & -0.0650 & 0.0042 & 0.4322 \\
Weight $(\mathrm{kg})$ & 0.0230 & 0.0005 & 0.7846 \\
BMI $\left(\mathrm{kg} / \mathrm{m}^{2}\right)$ & 0.0632 & 0.0040 & 0.7846 \\
Waist circumference $(\mathrm{cm})$ & 0.0270 & 0.0007 & 0.7444 \\
Muscle quantity $(\mathrm{kg})$ & -0.0008 & 0.0000 & 0.9920 \\
Body-fat quantity $(\mathrm{kg})$ & 0.0367 & 0.0013 & 0.6576 \\
Body-fat percentage $(\%)$ & 0.0398 & 0.0016 & 0.6314 \\
Hemoglobin A1c $(\%)$ & -0.1988 & 0.0395 & 0.0154 \\
Total cholesterol $(\mathrm{mg} / \mathrm{dl})$ & -0.0369 & 0.0014 & 0.6558 \\
HDL-cholesterol $(\mathrm{mg} / \mathrm{dl})$ & -0.0069 & 0.0069 & 0.9336 \\
LDL-cholesterol $(\mathrm{mg} / \mathrm{dl})$ & -0.0192 & 0.0004 & 0.8171 \\
Triglycerides $(\mathrm{mg} / \mathrm{dl})$ & -0.0849 & 0.0072 & 0.3048 \\
eGFR $\left(\mathrm{ml} / \mathrm{min} / 1.73 \mathrm{~m}{ }^{2}\right)$ & -0.2310 & 0.0533 & 0.0047 \\
\hline
\end{tabular}

$B M I$ body mass index, $L D L$ low-density lipoprotein, $H D L$ high-density lipoprotein, $e G F R$ estimated glomerular filtration rate showed a significantly negative relationship with changes in Fat $\%$.

\subsection{Simple and Multivariable Regression Analysis for Changes in Fat \% after Add-on SGLT2 Inhibitor Treatment}

Table 3 shows the results of multivariable regression analysis for changes in Fat \% after add-on SGLT2 inhibitor treatment. Pretreatment levels of $\mathrm{HbA} 1 \mathrm{c}$ and eGFR were significant and independent factors (pretreatment levels of HbA1c: $\beta=-0.1783, p=0.0319$; eGFR: $\beta=-0.2182$, $p=0.0072)$.

\subsection{Logistic Regression Analysis for Fat \% change after Add-on SGLT2 Inhibitor Treatment}

We defined the greater body fat reduction group as having a reduction of $>0.4 \%$ in Fat $\%$ (median) after add-on SGLT2 inhibitor treatment. Table 4 shows the results of the simple logistic regression analysis for greater body fat reduction. Pretreatment $\mathrm{HbAlc}$ levels as the continuous variable [odds ratio (OR) $1.54 ; 95 \%$ confidence interval (CI) $1.13-2.11, p<0.01]$ and smoking (OR 2.54; 95\% CI $1.18-5.47, p=0.02$ ) were significant factors for greater body fat reduction with SGLT2 inhibitors. The multivariate logistic regression analysis demonstrated that the pretreatment HbA1c levels (OR 1.61; 95\% CI 1.16-2.25; $p<0.01$ ) and smoking (OR 2.65; 95\% CI, 1.14-6.15; $p=0.02$ ) were significantly associated factors for greater body fat reduction with SGLT2 inhibitors.

\subsection{Receiver Operating Characteristic Curve Analysis of Pretreatment Levels of HbA1c}

We conducted an ROC analysis to calculate a cut-off value of pretreatment $\mathrm{HbA} 1 \mathrm{c}$ levels for the percentage decrease in greater body fat. The cut-off value of the pretreatment levels of HbA1c for the greater Fat \% change after SGLT2 inhibitors therapy was $7.7 \%$ (sensitivity $53 \%$ and specificity $69 \%, p<0.01$ ).

\subsection{Subgroup Analyses Regarding Pretreatment HbA1c Levels, GLP-1 Analog Treatment, Smoking Status, and Body Mass Index at Baseline for Appreciable Body Fat Reduction by SGLT2 Inhibitors}

We compared the percentage changes in body fat after addon SGLT2 inhibitor treatment between the higher HbA1c group (pretreatment levels of $\mathrm{HbAlc} \geq 7.7 \%$ ) and the lower HbA1c group (pretreatment levels of HbA1c $<7.7 \%$ ). Figure 2 shows that the reduction in 
Table 3 Results of multivariate regression analysis for body-fat percentage change after sodium-glucose co-transporter 2 inhibitor treatment

\begin{tabular}{|c|c|c|c|c|c|c|}
\hline & \multicolumn{2}{|c|}{ Unstandardized coefficients } & \multirow{2}{*}{$\begin{array}{l}\text { Standardized coefficients } \\
\beta\end{array}$} & \multicolumn{2}{|l|}{$95 \% \mathrm{CI}$ for $\mathrm{B}$} & \multirow[t]{2}{*}{$p$ value } \\
\hline & $B$ & SEM & & Lower bound & Upper bound & \\
\hline Constant & 0.0142 & 0.0156 & & -0.0167 & 0.0451 & 0.2373 \\
\hline Sex (male) & 0.0005 & 0.0025 & 0.0155 & -0.0044 & 0.0054 & 0.8473 \\
\hline Age (year) & 0.0001 & 0.0001 & 0.0622 & -0.0001 & 0.0003 & 0.4690 \\
\hline $\operatorname{BMI}\left(\mathrm{kg} / \mathrm{m}^{2}\right)$ & 0.0002 & 0.0003 & 0.0808 & -0.0003 & 0.0008 & 0.3352 \\
\hline Hemoglobin A1c (\%) & -0.0021 & 0.0010 & -0.1783 & -0.0040 & -0.0002 & 0.0319 \\
\hline eGFR $\left(\mathrm{ml} / \mathrm{min} / 1.73 \mathrm{~m}^{2}\right)$ & -0.0002 & 0.0001 & -0.2182 & -0.0003 & -0.0001 & 0.0072 \\
\hline
\end{tabular}

$R^{2}=0.0971 . F$ test for liner relationship was 3.0529 with $p=0.012$. Variance inflation factors (VIF is an index used to detect the multicollinearity between exploratory variables): sex 1.0101; age 1.1557; BMI 1.0973; Hemoglobin A1c 1.0649; eGFR 1.0062

$B M I$ body mass index, $e G F R$ estimated glomerular rate

Table 4 Results of logistic regression analysis for body-fat percentage change after sodiumglucose co-transporter 2 inhibitor treatment $(>0.4 \%)$

\begin{tabular}{|c|c|c|c|c|c|c|c|c|}
\hline \multirow[b]{3}{*}{ Sex (male) } & \multicolumn{4}{|c|}{ Simple regression } & \multicolumn{4}{|c|}{ Multivariate regression } \\
\hline & \multirow{2}{*}{$\frac{\mathrm{OR}}{1.4031}$} & \multicolumn{2}{|l|}{$95 \% \mathrm{CI}$} & \multirow{2}{*}{$\frac{p \text { value }}{0.3593}$} & \multirow{2}{*}{$\frac{\mathrm{OR}}{1.2038}$} & \multicolumn{2}{|l|}{$95 \% \mathrm{CI}$} & \multirow{2}{*}{$\frac{p \text { value }}{0.6444}$} \\
\hline & & 0.6801 & 2.8944 & & & 0.5477 & 2.6459 & \\
\hline Age (year) & 0.9954 & 0.9643 & 1.0275 & 0.7771 & 1.0194 & 0.9823 & 1.0579 & 0.3098 \\
\hline Height $(\mathrm{cm})$ & 1.0202 & 0.9807 & 1.0614 & 0.3206 & & & & \\
\hline Weight (kg) & 1.0050 & 0.9839 & 1.0266 & 0.6468 & & & & \\
\hline $\operatorname{BMI}\left(\mathrm{kg} / \mathrm{m}^{2}\right)$ & 1.0053 & 0.9359 & 1.0799 & 0.8840 & 1.0197 & 0.9384 & 1.1081 & 0.6449 \\
\hline Waist circumference $(\mathrm{cm})$ & 1.0224 & 0.9950 & 1.0505 & 0.1104 & & & & \\
\hline Muscle quantity (kg) & 1.0086 & 0.9719 & 1.0466 & 0.6510 & & & & \\
\hline Body-fat quantity $(\mathrm{kg})$ & 1.0051 & 0.9720 & 1.0394 & 0.7649 & & & & \\
\hline Body-fat percentage $(\%)$ & 1.0922 & 0.0193 & 61.8618 & 0.9658 & & & & \\
\hline Hypertension, $n(\%)$ & 0.7582 & 0.3945 & 1.4572 & 0.4062 & & & & \\
\hline Dyslipidemia, $n(\%)$ & 0.8949 & 0.3551 & 2.2553 & 0.8138 & & & & \\
\hline Current smoking, $n(\%)$ & 2.5417 & 1.1819 & 5.4657 & $0.0169^{*}$ & 2.6466 & 1.1396 & 6.1469 & $0.0236^{*}$ \\
\hline Hemoglobin A1c (\%) & 1.5419 & 1.1288 & 2.1061 & $0.0065^{* *}$ & 1.6090 & 1.1517 & 2.2480 & $0.0053^{* * *}$ \\
\hline Total cholesterol $(\mathrm{mg} / \mathrm{dl})$ & 1.0015 & 0.9926 & 1.0105 & 0.7391 & & & & \\
\hline HDL-cholesterol (mg/dl) & 0.9917 & 0.9641 & 1.0202 & 0.5656 & & & & \\
\hline LDL-cholesterol (mg/dl) & 1.0020 & 0.9912 & 1.0129 & 0.7222 & & & & \\
\hline Triglycerides (mg/dl) & 1.0009 & 0.9983 & 1.0035 & 0.5143 & & & & \\
\hline eGFR $\left(\mathrm{ml} / \mathrm{min} / 1.73 \mathrm{~m}^{2}\right)$ & 1.0092 & 0.9888 & 1.0300 & 0.3779 & & & & \\
\hline
\end{tabular}

Hosmer-Lemeshow goodness-of-fit $\chi^{2}$ was 12.84 with a $p$ value of 0.12

$B M I$ body mass index, $L D L$ low-density lipoprotein, $H D L$ high-density lipoprotein, $G F R$ estimated glomerular filtration rate

${ }^{*} P<0.05 ;{ }^{* *} P<0.01$
Fat $\%$ was significantly greater in the higher $\mathrm{HbA} 1 \mathrm{c}$ group than the lower $\mathrm{HbAlc}$ group (higher $\mathrm{HbAlc}$ group $-0.7 \pm 1.3 \%$ vs. lower HbAlc group $-0.1 \pm 1.4 \%$, $p=0.01$ ). However, the reduction in body weight was not significantly different among the two groups, as shown in Fig. 3. In other body composition results, the reduction in water (higher $\mathrm{HbAlc}$ group $-0.02 \pm 0.86 \mathrm{~kg}$ vs. lower HbAlc group $-0.56 \pm 0.94 \mathrm{~kg}, p<0.01)$ and skeletal muscle (higher HbA1c group $-0.01 \pm 1.09 \mathrm{~kg}$ vs. lower HbA1c group $-0.68 \pm 1.19 \mathrm{~kg}, \quad p=0.04) \quad$ was significantly greater in the lower HbA1c group than the higher $\mathrm{HbA1c}$ group. However, the reduction in fat (higher HbA1c group $-0.85 \pm 1.16 \mathrm{~kg}$ vs. lower HbA1c group $-0.46 \pm 1.19 \mathrm{~kg}, p=0.04)$ was significantly greater in the higher $\mathrm{HbA1c}$ group than the lower HbA1c group.

In this study, 13 patients were treated with combined GLP-1 receptor agonists, and these agents did not influence body composition change by SGLT-2 inhibitors (Fat \%: GLP-1 receptor agonists; $-0.4 \pm 1.4$, no GLP-1 receptor agonists; $-0.7 \pm 1.2, p=0.41$ ). 


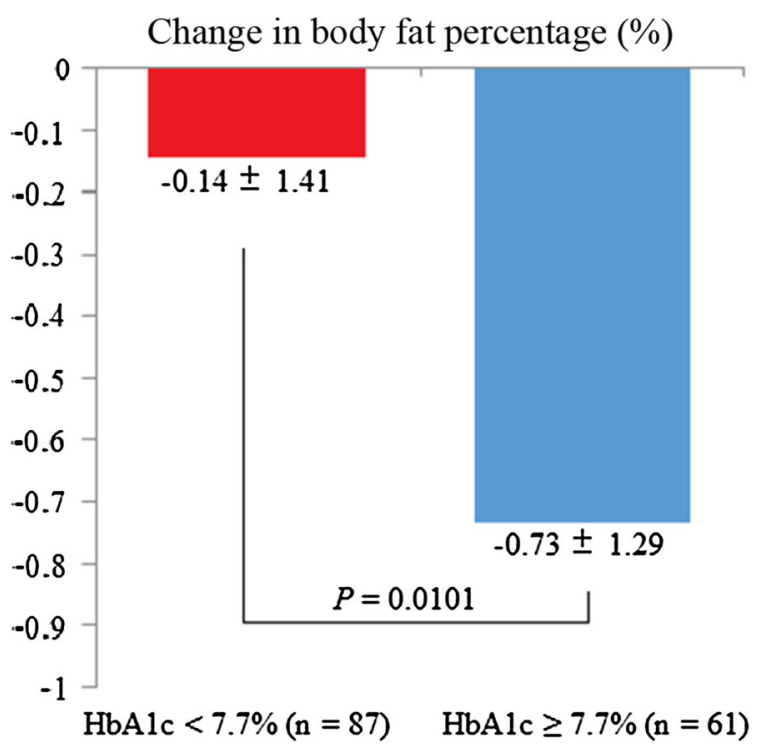

Fig. 2 Changes in Fat \% by add-on SGLT2 inhibitor treatment in patients with pretreatment HbA1c levels $<7.7 \%$ and those with pretreatment $\mathrm{HbA1c}$ levels $\geq 7.7 \%$. Data are mean \pm standard deviation. Fat $\%$ body fat percentage, SGLT2 sodium-glucose cotransporter 2, HbAlc glycated hemoglobin

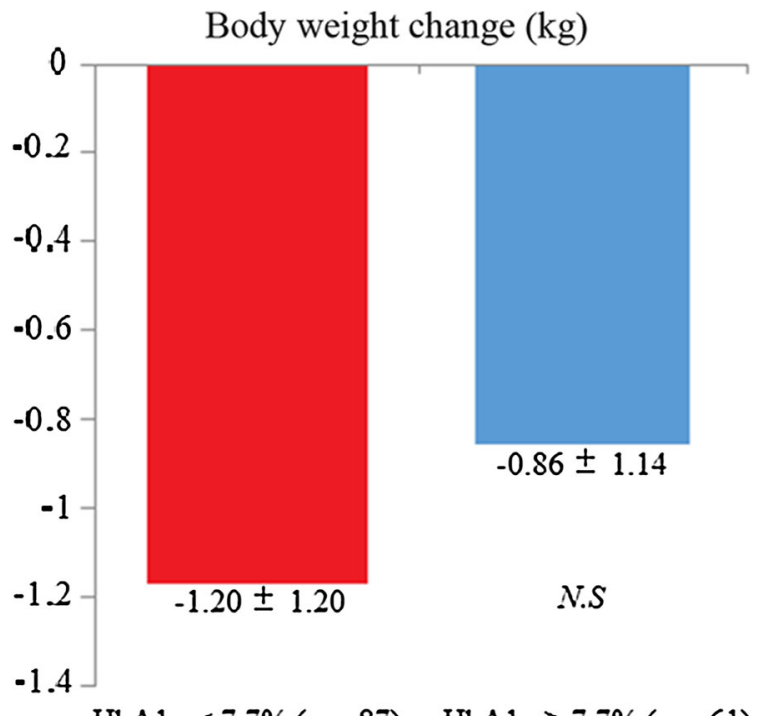

$\mathrm{HbAlc}<7.7 \%(\mathrm{n}=87) \quad \mathrm{HbAlc} \geq 7.7 \%(\mathrm{n}=61)$

Fig. 3 Changes in body weight as a result of add-on SGLT2 inhibitor treatment in patients with pretreatment $\mathrm{HbA} 1 \mathrm{c}$ levels $<7.7 \%$ and those with pretreatment HbA1c levels $\geq 7.7 \%$. Data are mean \pm standard deviation. SGLT2 sodium-glucose cotransporter 2, HbAlc glycated hemoglobin

We also compared the changes in the Fat \% after add-on SGLT2 inhibitor treatment between smokers and nonsmokers, and found that the Fat $\%$ was significantly reduced in the smoker group (smoker group $-0.8 \pm 1.3 \%$ vs. non-smoker group $-0.3 \pm 1.4 \%, p<0.05$ ).

A comparison of the changes in Fat $\%$ following add-on SGLT2 inhibitor treatment between obese (BMI $\geq 25 \mathrm{~kg}$ / $\mathrm{m}^{2}$ ) and non-obese $\left(\mathrm{BMI}<25 \mathrm{~kg} / \mathrm{m}^{2}\right)$ groups showed no significant difference between the two groups (obese group $-0.4 \pm 1.5 \%$ vs. non-obese group $-0.5 \pm 1.0 \%$, $p=0.69$ ).

The median levels of Fat $\%$ at pretreatment with SGLT2 inhibitors in men and women were 29.7 and 39.34\%, respectively. When we compared the changes in Fat \% following add-on SGLT2 inhibitor treatment between the excess fat group (over the median) and non-excess fat group (less than the median), there was no significant difference between the two (non-excess fat group $-0.5 \pm 1.3 \%$ vs. excess fat group $-0.3 \pm 1.5 \%, p=0.34$ ).

In obese patients with good diabetic control (BMI $\geq 25$ $\mathrm{kg} / \mathrm{m}^{2}$ and pre-treatment levels of HbA1c $<7.0 \%$ ), SGLT2 inhibitor therapy significantly decreased the levels of $\mathrm{HbA1c}$ (from $6.40 \pm 0.15 \%$ to $6.29 \pm 0.22 \%, p=0.03$ ). We found no significant changes in the quantity of body fat (from 27.88 to $27.49 \mathrm{~kg}, \mathrm{p}=0.14$ ) and Fat \% (from 35.38 to $35.29 \%, p=0.77$ ).

The multivariate logistic regression analysis with a nominal variable of the pretreatment $\mathrm{HbA1c}$ levels (higher $\mathrm{HbA} 1 \mathrm{c} \geq 7.7 \%$ and lower $\mathrm{HbA} 1 \mathrm{c}<7.7 \%$ ) also demonstrated that smoking and higher pretreatment levels of HbA1c $(\geq 7.7 \%)$ were independently and significantly correlated with the greater body fat reduction, as shown in Table 5.

In the non-smoker group, pretreatment levels of HbA1c were also independently and significantly correlated with greater body fat reduction as shown in Table 6 (OR 1.56; 95\% CI $1.05-2.37 ; p=0.03$ ).

\section{Discussion}

In the present study pretreatment levels of $\mathrm{HbAlc}$ and smoking status were significantly associated with SGLT2 inhibitor-induced greater reduction in Fat $\%$ in the initial 4 weeks of SGLT2 inhibitor therapy. The effective plasma glucose-lowering and weight-decreasing actions of SGLT2 inhibitors have previously been reported [9], and our results also demonstrated the significant body fat-reducing effects $(-0.62 \mathrm{~kg})$ of SGLT2 inhibitors even over a short treatment period (4 weeks). SGLT2 inhibitor users have been suggested to increase ketoacidosis risk [10, 11], but no patients became ketoacidosic during the present study.

It is reported that SGLT2 inhibitor therapy mainly reduces fat weight [9]. We found a significant correlation between the initial changes in total body weight and Fat \% through the 4-week add-on SGLT2 inhibitor treatment, but the relationship was not strong, indicating that the SGLT2 inhibitor-induced weight reduction was not necessarily associated with a reduction in body fat quantity. Body fat reduction is the favorable clinical goal for management of 

regression analysis for body fat percentage reduction, when we divided the patients into two groups by setting a cut-off value of $7.7 \%$ of glycated hemoglobin (HbA1c)
Table 5 Multivariate logistic

\begin{tabular}{lllll}
\hline & OR & \multicolumn{2}{l}{$95 \% \mathrm{CI}$} & $p$ value \\
\cline { 3 - 5 } & & Lower bound & Upper bound & \\
\hline Sex & 1.2384 & 0.5664 & 2.7076 & 0.5922 \\
Sex (male) & 1.0156 & 0.9796 & 1.0529 & 0.4002 \\
Age (year) & 1.0180 & 0.9396 & 1.1031 & 0.6621 \\
HbA1c $(\geq 7.7 \%$, yes) & 2.7300 & 1.3457 & 5.5384 & $0.0054^{*}$ \\
Current smoker (yes) & 2.4825 & 1.0831 & 5.6899 & $0.0317^{*}$ \\
\hline
\end{tabular}

$B M I$ body mass index, $O R$ odds ratio, $C I$ confidence interval

Hosmer-Lemeshow goodness-of-fit $\chi^{2}$ was 3.21 with $p$ value of 0.92

${ }^{*} P<0.05 ;{ }^{* * *} P<0.01$

\begin{tabular}{lllll}
\hline & OR & \multicolumn{2}{l}{$95 \% \mathrm{CI}$} & $p$ value \\
\cline { 3 - 5 } & & Lower bound & Upper bound & \\
\hline Sex (male) & 1.2144 & 0.5279 & 2.7939 & 0.6477 \\
Age (year) & 1.0272 & 0.9859 & 1.0702 & 0.2005 \\
BMI & 1.0043 & 0.9122 & 1.1056 & 0.9307 \\
Hemoglobin A1c (per 1.0\%) & 1.5778 & 1.0514 & 2.3678 & $0.0277^{*}$ \\
\hline
\end{tabular}

$B M I$ body mass index, $O R$ odds ratio, $C I$ confidence interval

Hosmer-Lemeshow goodness-of-fit $\chi^{2}$ was 6.2 with $p$ value of 0.625

${ }^{*} P<0.05 ;{ }^{* *} P<0.01$

patients with T2DM. In the present study, we evaluated SGLT2 inhibitor-induced changes in Fat \% for a good and practical indicator of body fat reduction considering the body composition balance. Because the cut-off value of Fat $\%$ for a greater response in fat reduction was not defined in the previous studies and we could not assess whether some Fat \% reduction was linked to substantial improvement of metabolic abnormalities, we applied the median value of Fat \% reduction after add-on SGLT2 inhibitor treatment in the present study; $-0.4 \%$ as the cutoff value. We defined a greater response in fat reduction following SGLT2 inhibitors as more than $0.4 \%$ reduction in Fat \%.

This study clearly showed that the SGLT2 inhibitor-induced change in Fat \% was significantly correlated with the pretreatment levels of $\mathrm{HbA} 1 \mathrm{c}$, and the additional administration of SGLT2 inhibitors to the uncontrolled hyperglycemic state effectively decreased Fat $\%$. In patients with T2DM, SGLT2 inhibitors evoke glycosuria, lowering blood glucose levels, and subsequently liver glyconeogenesis and glucagon secretion occur to prevent hypoglycemia $[12,13]$. Because the glucagon receptor is expressed in adipose tissue, the secreted glucagon enhances lipolysis and increases fatty acid release in adipose tissue. The excess energy is stored in white adipose tissue as triglyceride. Fatty acid and glycerol are emitted to maintain the energy balance of whole-body metabolism in a fasting state. It is known that the attenuated action of insulin strongly influences enhanced lipolysis, while the excess levels of glucagon have a weak effect on it. Because the glucose-lowering action of insulin is effective on the overall metabolic balance in a comparatively good glycemic state, the SGLT2 inhibitor-induced, counter-regulated increase in glucagon levels will lower its effects on the lipolysis of fat tissue. Based on these metabolic mechanisms, we considered that the uncontrolled diabetic condition with higher pretreatment levels of HbA1c effectively influences a considerable reduction in Fat \% by SGLT2 inhibitors.

In this study, we unexpectedly found that smoking is significantly correlated with changes in the Fat \% resulting from add-on SGLT2 inhibitor treatment. The detailed mechanism of smoking on SGLT2 inhibitor-induced fat reduction is presently unknown and this observation might be a chance one. However, in previous clinical observations, smoking decreased body weight while smoking cessation induced body weight gain [14], suggesting that smoking has some kind of influence on body composition, particularly with regard to body fat. It is possible that smoking exhibits enhanced lipolysis and fat-burning action on adipose tissue during hypoglycemia with SGLT2 inhibitors. Because smoking is an important risk factor for cardiovascular disease, instructions to stop smoking are essential for lifestyle management. Smoking might have a favorable effect on body fat reduction with add-on SGLT2 inhibitor treatment, but continuation of smoking is never clinically recommended. Further investigations are 
necessary to clarify the mechanisms of the effect of smoking with SGLT2 inhibitors on body fat reduction.

Metabolic syndrome increases the risk of cardiovascular diseases, and its main pathophysiology is insulin resistance. Our previous study showed that a muscle/fat ratio provided a good surrogate indicator of insulin resistance as assessed by the hyperinsulinemic-euglycemic clamp [13]. Thus, fat reduction is potentially a significant strategy for increasing insulin sensitivity in T2DM. Add-on SGLT2 inhibitor treatment itself improves insulin resistance [15] and reduces body-fat weight. The SGLT2 inhibitor-induced change in body composition with fat reduction is synergistically effective for the treatment of patients with metabolic syndrome and obesity. The body fat-reducing effect of SGLT2 inhibitors is stronger in visceral fats than in subcutaneous fat [16]. Therefore, introducing SGLT2 inhibitors will have additional benefits for the treatment of T2DM patients with obesity and insulin resistance.

In the ROC analysis, the cut-off value of pretreatment levels of HbA1c for a greater decrease in Fat \% of add-on SGLT2 inhibitor treatment was 7.7\%. The baseline BMI and the Fat $\%$ did not significantly correlate with the Fat $\%$ reduction. We demonstrated that the body fat-reducing action of SGLT2 inhibitors was not significantly effective in patients with better glucose control of $<7.7 \% \mathrm{HbA1c}$. In the normal animal model, SGLT2 inhibitors did not show significant weight reduction in non-diabetic mice fed a high-fat diet [17]. It seems that the administration of SGLT2 inhibitors offers additional benefits to patients with T2DM and a high HbA1c ( $\geq 7.7 \%)$ and is more effective for significantly reducing body-fat weight in patients with high HbA1c than in those with low HbA1c.

Our study had some limitations. The study was retrospective and did not include a control group. We did not measure fasting blood glucose levels in all participants. We did not compare body fat change with the dual-energy X-ray absorptiometry (DEXA) and other methods. However, we did compare body composition using bioelectrical impedance analysis with InBody770, which is a highly reproducible and reliable method for analyzing body composition in daily clinical practice $[7,8,18]$. As this was a retrospective study, we were not able to investigate various factors such as combined therapy, diet, and exercise, which might affect the results, or different drugs and their doses. Future prospective studies focusing on these points are needed to confirm our results. It has been reported that change in body weight composition with SGLT2 inhibitors differs when administered in combination with GLP-1 receptor agonists [19]. We did not find a significant effect of GLP-1 receptor agonists on changes in Fat $\%$ in the present study. Further studies with larger populations should be undertaken to determine whether combined hypoglycemic medications can affect the results.

\section{Conclusion}

Additional treatment with SGLT2 inhibitors effectively decreased Fat \% in patients with T2DM and higher HbA1c levels before SGLT2 inhibitor administration. Our results suggest a greater initial response in the reduction of Fat \% in response to SGLT2 inhibitor therapy in patients with diabetes with pretreatment levels of HbA1c more than $7.7 \%$. In patients with T2DM with obesity and poor blood glucose status, SGLT2 inhibitor therapy is clinically expected to improve blood glucose control and reduce Fat \%. Thus, the introduction of SGLT2 inhibitors is a practical, effective, and promising strategy to promote aggressive treatment for uncontrolled T2DM at an early stage of DM-therapy.

\section{Compliance with ethical standards}

Funding The authors received no specific financial support for this study.

Conflict of interest HJ. has received honoraria from Novo Nordisk, Sanofi, AstraZeneca Pharmaceuticals, Astellas Pharma, Boehringer Ingelheim, Daiichi-Sankyo, Eli Lilly, Takeda, and Novartis Pharmaceuticals. S.S. has received honoraria from MSD, AstraZeneca Pharmaceuticals, Itamar Medical, Ono Pharmaceutical, and Novo Nordisk. There are no other potential conflicts of interest relevant to this article.

Ethical standards All procedures followed were in accordance with the ethical standards of the responsible committee on human experimentation (institutional and national) and with the Helsinki Declaration of 1964 and later versions. The Human Ethics Review Committee of Jinnouchi Hospital approved the study protocol. The protocol approval number of this study is $2016-8-3$ in our institution. Informed consent or a substitute for it was obtained from all patients included in the study.

Open Access This article is distributed under the terms of the Creative Commons Attribution-NonCommercial 4.0 International License (http://creativecommons.org/licenses/by-nc/4.0/), which permits any noncommercial use, distribution, and reproduction in any medium, provided you give appropriate credit to the original author(s) and the source, provide a link to the Creative Commons license, and indicate if changes were made.

\section{References}

1. Rabe K, Lehrke M, Parhofer KG, et al. Adipokines and insulin resistance. Mol Med. 2008;14:741-51.

2. Nauck MA, Del Prato S, Meier JJ, et al. Dapagliflozin versus glipizide as add-on therapy in patients with type 2 diabetes who have inadequate glycemic control with metformin: a randomized, 52-week, double-blind, active-controlled noninferiority trial. Diabetes Care. 2011;34:2015-22.

3. Vasilakou D, Karagiannis T, Athanasiadou E, et al. Sodiumglucose cotransporter 2 inhibitors for type 2 diabetes: A systematic review and meta-analysis. Ann Intern Med. 2013;159:262-74. 
4. Bolinder J, Ljunggren Ö, Kullberg J, et al. Effects of dapagliflozin on body weight, total fat mass, and regional adipose tissue distribution in patients with type 2 diabetes mellitus with inadequate glycemic control on metformin. J Clin Endocrinol Metab. 2012;97:1020-31.

5. Leiter LA, Yoon K-H, Arias P, et al. Canagliflozin provides durable glycemic improvements and body weight reduction over 104 weeks versus glimepiride in patients with type 2 diabetes on metformin: a randomized, double-blind, phase 3 study. Diabetes Care. 2015;38:355-64.

6. Yamada K, Nakayama H, Yoshinobu S, et al. Effects of a sodium glucose co-transporter 2 selective inhibitor, ipragliflozin, on the diurnal profile of plasma glucose in patients with type 2 diabetes: A study using continuous glucose monitoring. J Diabetes Investig. 2015;6:699-707.

7. Cha K, Chertow GM, Gonzalez J, Lazarus JM, Wilmore DW. Multifrequency bioelectrical impedance estimates the distribution of body water. J Appl Physiol. 1995;79:1316-9.

8. Cha K, Shin S, Shon C, Choi S, Wilmore DW. Evaluation of segmental bioelectrical impedance analysis (SIBA) for measuring muscle distribution. J Ichper Sd-Asia. 1997;11-4.

9. Bolinder J, Ljunggren Ö, Johansson L, et al. Dapagliflozin maintains glycaemic control while reducing weight and body fat mass over 2 years in patients with type 2 diabetes mellitus inadequately controlled on metformin. Diabetes Obes Metabol. 2014;16:159-69.

10. Hayami T, Kato Y, Kamiya H, et al. Case of ketoacidosis by a sodium-glucose cotransporter 2 inhibitor in a diabetic patient with a low-carbohydrate diet. J Diabetes Invest. 2015;6(5):587-90.

11. Ogawa W, Sakaguchi K. Euglycemic diabetic ketoacidosis induced by SGLT2 inhibitors: possible mechanism and contributing factors. J Diabetes Invest. 2016;7(2):135-8.
12. Ferrannini E, Muscelli E, Frascerra S, et al. Metabolic response to sodium-glucose cotransporter 2 inhibition in type 2 diabetic patients. J Clin Invest. 2014;124:199-508.

13. Merovci A, Solis-Herrera C, Daniele G, et al. Dapagliflozin improves muscle insulin sensitivity but enhances endogenous glucose production. J Clin Invest. 2014;124:509-14.

14. Aubin HJ, Farley A, Lycett D, Lahmek P, Aveyard P. Weight gain in smokers after quitting cigarettes: meta-analysis. BMJ. 2012;345:e4439.

15. Kurinami N, Sugiyama S, Yoshida A, et al. Correlation of body muscle/fat ratio with insulin sensitivity using hyperinsulinemiceuglycemic clamp in treatment-naïve type 2 diabetes mellitus. Diabetes Res Clin Pract. 2016;120:65-72.

16. Cefalu WT, Leiter LA, Yoon K-H, et al. Efficacy and safety of canagliflozin versus glimepiride in patients with type 2 diabetes inadequately controlled with metformin (CANTATA-SU): 52 week results from a randomised, double-blind, phase 3 non-inferiority trial. Lancet. 2013;382:941-50.

17. Komiya C, Tsuchiya K, Miyachi Y, et al. Ipragliflozin improves hepatic steatosis in obese mice and liver dysfunction in type 2 diabetic patients irrespective of body weight reduction. PLoS One. 2016;. doi:10.1371/journal.pone.0151511.

18. Bedogni G, Malavolti M, Severi S, et al. Accuracy of an eightpoint tactile-electrode impedance method in the assessment of total body water. Eur J Clin Nutr. 2002;56:1143-8.

19. Yutaka S, Daisuke Y, Takashi S, et al. Sodium-glucose cotransporter-2 inhibitor luseogliflozin added to glucagon-like peptide 1 receptor agonist liraglutide improves glycemic control with bodyweight and fat mass reductions in Japanese patients with type 2 diabetes: A 52-week, open-label, single-arm study. J Diabetes Invest. 2017. doi:10.1111/jdi.12694. 